\title{
miRNA-34a enhances the sensitivity of gastric cancer cells to treatment with paclitaxel by targeting E2F5
}

\author{
LINA LI ${ }^{1}$, CUILING WU ${ }^{2}$ and YUE ZHAO ${ }^{3}$ \\ Departments of ${ }^{1}$ Pathology and ${ }^{2}$ Biochemistry, Changzhi Medical College; ${ }^{3}$ Department of Dermatology, \\ Changzhi Heping Hospital Affiliated to Changzhi Medical College, Changzhi, Shanxi 046000, P.R. China
}

Received November 27, 2015; Accepted January 26, 2017

DOI: $10.3892 / \mathrm{ol} .2017 .6041$

\begin{abstract}
Gastric cancer (GC) is one of the most common types of malignant cancer worldwide, however improvements are required to the current therapies for GC. Although paclitaxel is one of the most promising chemotherapeutic agents in clinical use for GC, the resistance to paclitaxel that develops during treatment is a major obstacle to further treatments of GC. The present study reports that micro (mi) RNA-34a, a tumor suppressor in various types of cancer, may be an important regulator of chemoresistance in GC, as miRNA-34a mimics and inhibitors, enhance and inhibit the chemotherapeutic efficacy of paclitaxel, respectively. In addition, the present study identified that E2F transcription factor 5 (E2F5), a key oncogenic protein, is the direct target candidate of miRNA-34a. Previous studies have demonstrated that the inhibition of E2F5 by specific E2F5 small interfering RNA also increases the sensitivity of GC cells to paclitaxel. In conclusion, the present data suggest that miRNA-34a enhances the treatment of sensitive GC cells to paclitaxel by targeting E2F5. Therefore, the miRNA-34a/E2F5 axis appears to be a potential promising therapeutic target for overcoming the chemotherapeutic resistance of GC.
\end{abstract}

\section{Introduction}

Gastric cancer (GC) is one of the most common types of malignant cancer worldwide due to its high rates of morbidity and mortality (1). However, an effective treatment method for patients with GC is lacking, and improvements to the clinical outcome remains a challenge. Therefore, additional investigations into the underlying molecular mechanisms for the development and progression of GC, and the development of effective treatments for patients with GC is essential.

Correspondence to: Dr Yue Zhao, Department of Dermatology, Changzhi Heping Hospital Affiliated to Changzhi Medical College, 110 South Yanan Road, Changzhi, Shanxi 046000, P.R. China E-mail: zhaoyue8195@126.com

Key words: microRNA-34a, E2F transcription factor 5, paclitaxel, gastric cancer
Paclitaxel is a commonly used first-line chemotherapeutic drug for treatment of numerous types of cancer including breast (2), lung (3), colon (4), gastric (5) and cervical cancer (6) by regulating tubulin polymerization, inhibiting cell cycle progression and promoting apoptosis. However, cancer cells appear to be insensitive to paclitaxel following long-term treatment due to the acquisition of chemotherapeutic resistance (7). Thus, the inhibition of cancer chemoresistance to paclitaxel may significantly improve paclitaxel chemotherapy for various cancers.

microRNAs (miRNAs) are a group of small noncoding RNAs that negatively regulate target genes via either translational repression or mRNA degradation (8). An increasing number of studies have suggested that miRNAs perform a crucial role in regulating cancer cells by acting as oncogenes or tumor suppressers (9-11). Therefore, miRNAs may serve as potential biomarkers and targets for the diagnosis and treatment of various types of cancer (12). miRNA-34a is an important regulator of tumor suppression, and acts by controlling cellular proliferation, cellular differentiation and the survival of cancer cells (13). A previous study demonstrated that the expression of miRNA-34a in GC was markedly downregulated, and that miRNA-34a was able to significantly inhibit epidermal growth factor receptor (EGFR)-signaling-dependent matrix metalloproteinase-7 (MMP-7) activation, which suggested that miRNA-34a may serve as a tumor suppresser to inhibit the cellular proliferation, migration and invasion of GC cells (14). However, the role of miRNA-34a in GC remains poorly understood.

To investigate whether miRNA-34a may enhance the anticancer effect of paclitaxel on GC, the present study treated human GC cancer cells with miRNA-34a combined with paclitaxel. The results suggested that the introduction of miRNA-34a mimics and the miRNA-34a inhibitor into GC cells is able to enhance and decrease the chemotherapeutic efficacy of paclitaxel, respectively. Additional studies identified that E2F transcription factor 5 (E2F5) was a potential target of miRNA-34a. The results of the present study may increase the understanding of miRNA-34a in GC and facilitate the development of potential chemotherapeutic against GC.

\section{Materials and methods}

Cell culture. Human gastric cancer MKN45 and BGC823 cell lines were purchased from the Type Culture Collection of the 
Chinese Academy of Sciences (Shanghai, China). Cells were cultured in Dulbecco's modified Eagle's medium (DMEM; Invitrogen; Thermo Fisher Scientific, Inc., Waltham, MA, USA) containing 10\% fetal bovine serum (Gibco; Thermo Fisher Scientific, Inc.) and antibiotics (100 $\mathrm{U} \mathrm{ml}^{-1}$ penicillin and $100 \mu \mathrm{g} \mathrm{ml}^{-1}$ streptomycin), and maintained at $37^{\circ} \mathrm{C}$ in a humidified cell incubator with $5 \% \mathrm{CO}_{2}$ and $95 \%$ air. Paclitaxel (Sigma-Aldrich, Merck KGaA, Darmstadt, Germany) was dissolved prior to use at concentration of $0.1 \mathrm{mM}$ in DMEM, stored at $4^{\circ} \mathrm{C}$, and further diluted in DMEM to obtain each final concentration.

Cell transfection. $100 \mathrm{nM}$ of miRNA-34a mimic, miRNA-34a inhibitor or negative control (NC) miRNA-scrambled (SCR; Genepharma, Inc., Guangzhou, Guangdong, China), E2F5-specific siRNA or negative control (NC) siRNA (Shanghai Shengong Biological Engineering Technology \& Services, Ltd., Shanghai, China) or $2 \mu \mathrm{g}$ of pmirGLO-E2F5 or pmirGLO-MutE2F5 reporter constructs were transfected into MKN45 or BGC823 cells at $70 \%$ confluence using Lipofectamine $^{\mathrm{TM}} 2000$ (Thermo Fisher Scientific, Inc.), according to the manufacturer's protocol. The siRNA sequences were as follows: NC siRNA, CATGTCATGTGTCACATCTC; E2F5-siRNA, CAGGACCTATCCATGTGCTGCTTAT. The miRNA sequences were not provided by the suppliers, in order to protect commercial secrets.

3'-untranslated region (UTR) luciferase reporter assay. Potential target genes for miRNA-34a were identified using TargetScan (http://www.targetscan.org) software, and E2F transcription factor 5 protein (E2F5) was identified as a target candidate of interest.

The genomic DNA of MKN45 cells was isolated using a QIAamp UCP DNA Micro Kit (Qiagen, Inc., Valencia, CA, USA). The 3'-UTR sequence of the human E2F5 gene containing the miRNA-34a binding site was generated by polymerase chain reaction (PCR) with LA Taq DNA polymerase (Takara, Dalian, China) using a Eppendorf Mastercycler nexus Thermal Cycler (Eppendorf, Hamburg, Germany) with conditions as follows: Initial denaturation at $95^{\circ} \mathrm{C}$ for $5 \mathrm{~min}$, followed by 33 cycles of $60 \mathrm{sec}$ at $95^{\circ} \mathrm{C}$ for denaturing, $30 \mathrm{sec}$ at $60^{\circ} \mathrm{C}$ for annealing and $60 \mathrm{sec}$ at $72^{\circ} \mathrm{C}$ for extension, then a final extension at $72^{\circ} \mathrm{C}$ for $10 \mathrm{~min}$. PCR was performed with the following primers: E2F5 UTR forward, CGGCTAGCTTCTGGATTTCAACTTTTCTTC; E2F5 UTR reverse, GCGTCGACGGAAAGTGGAATGTCAGAAGTC (Shanghai Shengong Biological Engineering Technology \& Services, Ltd.). The 3'-UTR was subsequently cloned into the pmirGLO-report vector (Promega Corporation, Madison, WI, USA), which was denoted as pmirGLO-E2F5.

The mutated (Mut) E2F5 3'-UTR miRNA-34a binding sites oligonucleotide was generated by deletion mutagenesis with overlapping extension PCR. Initially, two parallel PCRs were conducted with pmirGLO-E2F5 as the template: One reaction was performed with the previously described E2F5 UTR forward primer and a Mut E2F5 UTR reverse primer with the sequence, ACTTTACAAGTGAACGTCTGT; the other PCR was performed with a Mut forward miRNA-34a binding site primer with the sequence AACAGACGTTCCACTTGTAA and the previously described E2F5 UTR reverse primer
(Shanghai Shengong Biological Engineering Technology \& Services, Ltd.). A total of $10 \mu \mathrm{l}$ equimolar mixture of the two PCR products was used as a template with the E2F5 UTR forward and reverse primers to obtain a Mut 3'-UTR of E2F5 oligonucleotide. The Mut 3'-UTR oligonucleotide was subsequently cloned into the pmirGLO-report vector, designated as pmirGLO-MutE2F5. All constructs were sequenced by Shanghai Shengong Biological Engineering Technology \& Services, Ltd.

For the luciferase reporter assay, $3 \times 10^{5} \mathrm{MKN} 45$ or BGC823 GC cells were seeded in each well of 24-well plates and co-transfected with the pmirGLO-E2F5 or pmirGLO-MutE2F5 reporter constructs, and miRNA-34a mimics, miRNA-34a inhibitors or miRNA-SCR, using Lipofectamine $2000^{\mathrm{TM}}$ (Thermo Fisher Scientific, Inc.). Luciferase activities were determined using a Dual-Luciferase ${ }^{\circledR}$ Reporter assay system (Promega Corporation). Data were normalized and presented as the firefly/Renilla luciferase ratio.

Western blot analysis. Following transfection for $24 \mathrm{~h}$, cells were lysed using cell lysis buffer (2\% SDS, 6 M urea, $200 \mathrm{mM}$ ammonium bicarbonate, $0.1 \%$ protease inhibitor cocktail). Subsequent to the quantification of cell lysate protein concentration with a Bicinchoninic Acid Protein Assay kit (Pierce; Thermo Fisher Scientific, Inc.) according to the manufacturer's protocol, $30 \mu \mathrm{g}$ of cell lysate in each lane was separated with 10\% SDS-PAGE and transferred onto nitrocellulose membranes (Bio-Rad Laboratories, Inc., Hercules, CA, USA). The membranes were blocked with skimmed dried milk in PBS buffer for $1 \mathrm{~h}$ at room temperature, and immunoblotted using primary antibodies against E2F5 (dilution, 1:1,000; cat no. sc-1082; Santa Cruz Biotechnology, Inc.) or $\beta$-actin (dilution, 1:5,000; cat. no. A3854; Sigma-Aldrich; Merck KGaA) at $4{ }^{\circ} \mathrm{C}$ overnight. Membranes were then incubated with the horseradish peroxidase-conjugated secondary antibody (dilution, 1:2,000; cat no. M21002; Abmart, Shanghai, China) at room temperature for $1 \mathrm{~h}$. Immunolabeling was detected using $100 \mathrm{ml}$ Luminata Forte Western HRP substrate (EMD Millipore, Billerica, MA, USA), followed by exposure to film. The relative intensity of the bands was quantified using ImageJ software version 1.41 (NIH, Bethesda, MD, USA).

Reverse transcription-quantitative PCR (RT-qPCR). Total RNA was extracted from MKN28 and BGC823 cells using TRIzol reagent (Invitrogen; Thermo Fisher Scientific, Inc.), according to the manufacturer's protocol. Reverse transcription was performed to produce complementary (c) DNA with $1 \mu \mathrm{g}$ RNA incubated with the specific primers and reaction buffer of the Superscript system (Invitrogen; Thermo Fisher Scientific, Inc.) at $16^{\circ} \mathrm{C}$ for $25 \mathrm{~min}, 42^{\circ} \mathrm{C}$ for $30 \mathrm{~min}$ and $85^{\circ} \mathrm{C}$ for 5 min. PCR primers for miRNA-34a and U6 RNA were purchased from GeneCopoeia, Inc. (Guangzhou, China). The following PCR primers were used in qPCR: E2F5 forward, 5'-CCTGTTCCCCCACCTGATG-3' and reverse, 5'-TTTCTG TGGAGTCACTGGAGTCA-3'; and $\beta$-actin forward, 5'-CTG GAACGGTGAAGGTGACA-3' and reverse, 5'-AAGGGA CTTCCTGTAACAATGCA-3'. Primers were synthetized by Shanghai ShengGong Biology Engineering Technology Service, Ltd. (Shanghai, China). miRNA-34a expression was determined using Hairpin-it TM miRNAs qPCR kit 
(Shanghai GenePharma Co., Ltd., Shanghai, China). U6 RNA was used as an endogenous control. The mRNA levels of E2F5 and $\beta$-actin were detected using the SYBR green PCR kit (Applied Biosystems; Thermo Fisher Scientific, Inc.). qPCR was performed on an ABI-7500 PCR system (Applied Biosystems; Thermo Fisher Scientific, Inc.). Quantitative PCR thermocycling conditions were: $95^{\circ} \mathrm{C}$ for $10 \mathrm{~min}$ initially, followed by 35 cycles of $95^{\circ} \mathrm{C}$ for $15 \mathrm{sec}$, and $60^{\circ} \mathrm{C}$ for $45 \mathrm{sec}$. Data were analyzed using the $2^{-\Delta \Delta \mathrm{Cq}}$ method, as previously described (15).

MTT assay. MKN45 and BGC823 cells were plated in 96-well plates at a density of $5 \times 10^{3}$ cells/well and cultured at $37^{\circ} \mathrm{C}$ for $18 \mathrm{~h}$. Following transfection with miR-SCR or miR-34a inhibitor for $24 \mathrm{~h}$, followed by treatment with $100 \mathrm{nM}$ paclitaxel for another $16 \mathrm{~h}$, cellular viability was determined using the MTT assay. Following this treatment for $16 \mathrm{~h}, 5 \mu \mathrm{g} / \mathrm{ml}$ of MTT (Sigma-Aldrich; Merck KGaA, Darmstadt, Germany) was added into each well, and incubated at $37^{\circ} \mathrm{C}$ for $2 \mathrm{~h}$. The supernatant was then removed, and $100 \mu 1$ DMSO was added into each well to dissolve the formazan product. The optical density at wavelength of $570 \mathrm{~nm}$ was determined using the ELx808 absorbance reader (BioTek Instruments, Inc., Winooski, VT, USA).

5-Bromo-2-deoxyUridine (BrdU) assay. The BrdU incorporation assay kit (Roche Applied Science, Penzberg, Germany) was used for analyzing the incorporation of BrdU during DNA synthesis in proliferating cells, according to the manufacturer's protocol. A total of $2 \times 10^{3} \mathrm{MKN} 28$ cells were cultured for 24 or $48 \mathrm{~h}$, followed by incubation at $37^{\circ} \mathrm{C}$ for $1 \mathrm{~h}$ with $10 \mu \mathrm{M} \mathrm{BrdU}$ (BD Pharmingen, San Diego, CA, USA). The absorbance values were measured at a wavelength of $450 \mathrm{~nm}$ with the ELx808 plate reader.

Statistical analysis. Each experiment at least was performed 3 times. Data are presented as the mean \pm standard deviation. SPSS 18.0 software (SPSS, Inc., Chicago, IL, USA) was used to conduct statistical analyses. Multiple comparisons were analyzed using one-way analysis of variance followed by Tukey-Kramer post hoc analysis to test for differences between all groups. $\mathrm{P}<0.05$ was considered to indicate a statistically significant difference.

\section{Results}

miRNA-34a mimics enhance the chemotherapeutic efficacy of paclitaxel. As miRNA-34a is able to prevent metastasis in GC (14), it was hypothesized that miRNA-34a may enhance the efficacy of paclitaxel for the treatment of GC. MTT and BrdU assays were used to detect the cellular proliferation of GC cells, including MKN28 and BGC823 cells, which were transfected with miRNA-34a mimics or scrambled miRNA for $24 \mathrm{~h}$, followed by treatment with paclitaxel for another 16 h. qPCR demonstrated that transfection with miRNA-34a mimics resulted in a significant increase $(\mathrm{P}<0.01)$ of miRNA-34a in MKN28 and BGC823 cells compared with the cells transfected with the scrambled miRNA (Fig. 1A). The results of MTT assays demonstrated that the growth rate of GC cells transfected with miRNA-34a mimics was decreased compared with the cell transfected with the scrambled miRNA, and the growth rate of GC co-treated with miRNA-34a and paclitaxel was significantly decreased $(\mathrm{P}<0.05)$ compared with the cell transfected with miRNA-34a mimics alone (Fig. 1B). In addition, the BrdU assays also demonstrated similar results, as co-treatment with miRNA-34a and paclitaxel led to slower growth rate of GC compared with miRNA-34a mimics (Fig. 1C). These results demonstrated that transfection of miRNA-34a into GC enhances the inhibition of proliferation of GC cells by paclitaxel.

miRNA-34a inhibitors decrease the chemotherapeutic efficacy of paclitaxel. The present study also determined whether endogenous miRNA-34a inhibition by specific miRNA-34a inhibitors would decrease the chemotherapeutic efficacy of paclitaxel. The specific miRNA-34a inhibitors or scrambled miRNA-34a were transfected into GC cells for $24 \mathrm{~h}$, and followed by treatment with paclitaxel for another $16 \mathrm{~h}$. As expected, the introduction of miRNA-34a inhibitors significantly reduced the expression of miRNA-34a in MKN28 and BGC823 cells (Fig. 2A). The results of MTT and BrdU assays demonstrated that treatment with paclitaxel and miR-SCR resulted in a significant inhibition $(\mathrm{P}<0.05)$ of growth rate of GC cells compared with miR-SCR alone, and transfection miRNA-34a significantly attenuated the inhibitive effect of paclitaxel on the growth rate of GC cells (Fig. 2B and C). Therefore, these results indicate that miRNA-34a is involved in the chemotherapeutic efficacy of paclitaxel.

E2F5 is a direct target of miRNA-34a. TargetScan was used to identify the potential targets of miRNA-34a. As demonstrated in Fig. 3A, E2F transcription factor 5 protein (E2F5) was identified as an interesting candidate, as E2F5 has previously been observed as overexpressed in various types of cancer, including breast (16), epithelial ovarian (17), prostate cancer (18) and hepatocellular carcinoma (19), and is involved in cellular proliferation, cell growth and cell cycle progression. To investigate whether miRNA-34a is capable of regulating E2F5 expression in GC cells, qPCR was used. The present results demonstrated that transfection of scramble miRNA into GC MKN28 and BGC823 cells had no observed effect on E2F5 mRNA. However, miRNA-34a mimics significantly inhibited E2F5 mRNA, but miRNA-34a inhibitors significantly upregulated E2F5 mRNA (Fig. 3B). Additionally, western blot analysis also identified similar results as transfection of miRNA-34a mimics and miRNA-34a inhibitors into MKN28 cells could significantly repress and increase E2F5 protein levels, respectively (Fig. 3C).

To additionally investigate whether miRNA-34a negatively regulates E2F5 gene in GC by directly binding to the specific miRNA-34a binding site in the 3'UTR of E2F5 gene, two luciferase reporter recombinant vectors that contain either wild-type (Wt) 3'UTR of E2F5 gene (pmirGLO-E2F5) or a Mut E2F5 with mutated miRNA-34a binding sites (pmirGLO-MutE2F5) were constructed. The luciferase reporter assays in MKN28 cells demonstrated that miRNA-34a mimics and inhibitors significantly decreased and increased the luciferase activity of Wt E2F5 reporter (pmirGLO-E2F5), respectively. However, miRNA-34a and inhibitors had no effect on the luciferase activity of E2F5 Mut reporter (pmirGLO-MutE2F5) (Fig. 3D). 
A

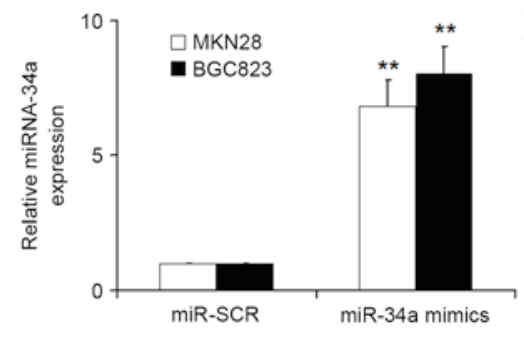

B

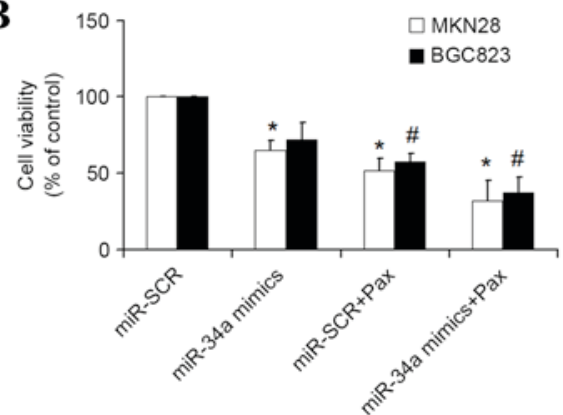

C

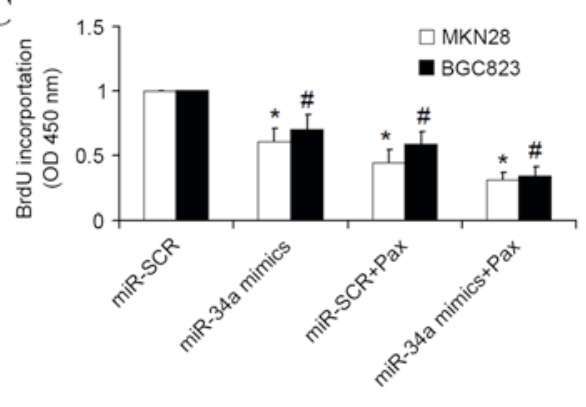

Figure 1. Overexpression of miR-34a in GC enhanced the inhibitory effect of paclitaxel on cellular proliferation. (A) Quantitative polymerase chain reaction was used to detect miR-34a expression levels in MKN28 and BGC823 cells transfected with either miR-34a mimics or miR-SCR (negative control). (B) MTT assays were used to determine the cell viability in MKN28 and BGC823 cells transfected with miR-SCR or miR-34a for 24 h, followed by treatment of paclitaxel $(100 \mathrm{nM})$ for another $16 \mathrm{~h}$. (C) BrdU incorporations were used to detect the cellular proliferation in MKN28 and BGC823 cells transfected with miR-SCR or miR-34a for $24 \mathrm{~h}$, followed by treatment of paclitaxel $(100 \mathrm{nM})$ for another $16 \mathrm{~h} .{ }^{*} \mathrm{P}<0.05$ vs. miR-SCR transfected MKN28 cells; ${ }^{*} \mathrm{P}<0.05 \mathrm{vs}$. miR-SCR transfected BGC823 cells; ${ }^{* *} \mathrm{P}<0.01$ vs. miR-SCR transfected cells. miR, microRNA; GC, gastric cancer; SCR, scrambled; BrdU, 5-Bromo-2-deoxyUridine; Pax, paclitaxel; OD, optical density.

A

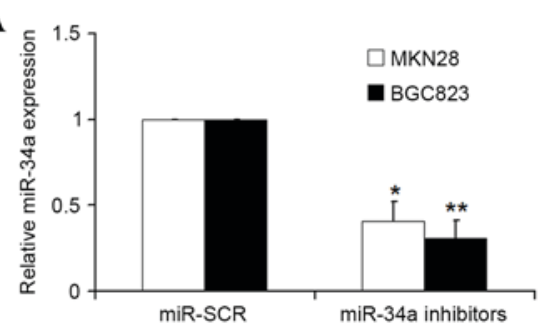

B

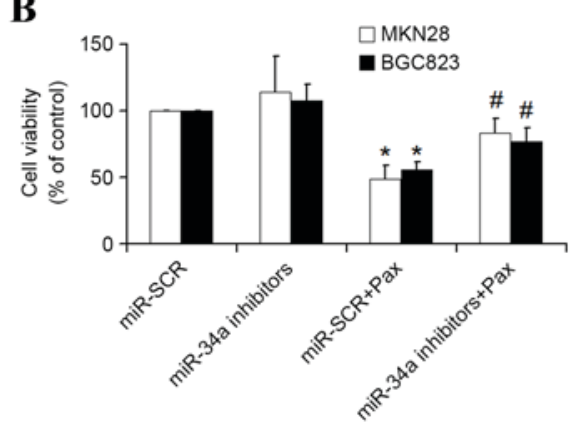

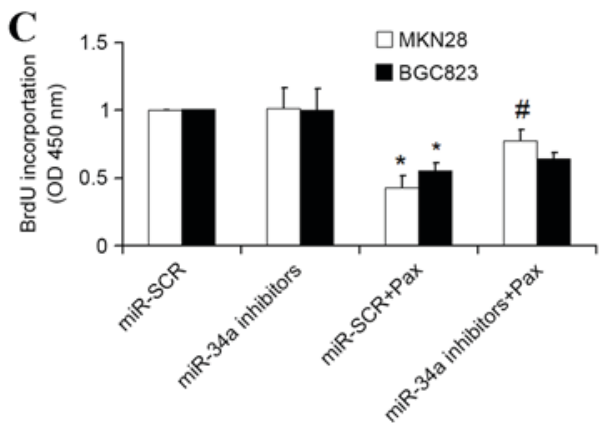

Figure 2. Overexpression of miR-34a inhibitors in GC attenuated the inhibitory effect of paclitaxel on cellular proliferation. (A) Quantitative polymerase chain reaction was used to detect miR-34a expression levels in MKN28 and BGC823 cells transfected with either miR-34a inhibitors or miR-SCR (negative control). (B) MTT assays were used to determine the cell viability in MKN28 and BGC823 cells transfected with miR-SCR or miR-34a inhibitors for 24 h, followed by treatment of paclitaxel $(100 \mathrm{nM})$ for another $16 \mathrm{~h}$. (C) BrdU incorporations were used to detect the cellular proliferation in MKN28 and BGC823 cells transfected with miR-SCR or miR-34a inhibitors for $24 \mathrm{~h}$, followed by treatment of paclitaxel (100 nM) for another $16 \mathrm{~h}$. * $\mathrm{P}<0.05 \mathrm{vs}$. miR-SCR transfected MKN28 cells; ${ }^{\#} \mathrm{P}<0.05$ vs. miR-SCR+paclitaxel treated BGC823 cells; ${ }^{* *} \mathrm{P}<0.01$ vs. miR-SCR transfected cells. miR, microRNA; GC, gastric cancer; SCR, scrambled; BrdU, 5-Bromo-2-deoxyUridine; Pax, paclitaxel; OD, optical density.

Overall, these results suggested that E2F5 is a direct target of miRNA-34a in GC cells.

E2F5 gene knockdown enhance the chemotherapeutic efficacy of paclitaxel. The above results demonstrated that miRNA-34a is able to increase the chemotherapeutic efficacy of paclitaxel, and that E2F5 is directly targeted by miRNA-34a. The present study therefore then investigated whether E2F5 knockdown by specific E2F5 small interfering (si) RNA can also enhance the chemotherapeutic efficacy of paclitaxel. Western blot analysis demonstrated that E2F5 siRNA but not NC siRNA overexpression decreased E2F5 protein level (Fig. 4A). MTT and BrdU assays demonstrated that E2F5 knockdown by specific siRNA is capable of significantly increasing the sensitivity of paclitaxel compared with NC siRNA, and that E2F5 overexpression results in a significant attenuation of the chemotherapeutic efficacy of paclitaxel (Fig. 4B and C). Therefore, E2F5 is also an important gene that involved in the chemotherapeutic resistance of paclitaxel, and E2F5 gene knockdown sensitized the GC to paclitaxel-induced proliferation inhibition.

\section{Discussion}

In the present study, the effect of miRNA-34a on the chemotherapeutic efficacy of paclitaxel was investigated. It was observed that miRNA-34a significantly enhanced the inhibitory role of paclitaxel in the proliferation of GC cells. In addition, the present study identified that miRNA-34a was able to directly target the E2F5 gene, which was demonstrated to be involved in the chemotherapeutic resistance of paclitaxel.

An increasing number of studies have suggested that miRNAs are a class of small highly conserved molecules that perform a crucial role in the regulation of multiple cellular events including cellular proliferation, differentiation, development and apoptosis $(8,20,21)$. Dysregulation and dysfunction of miRNAs leads to the initiation and development of cancers, indicating that miRNAs are important molecules that can potentially be used for the diagnosis and treatment of cancers (22). miRNA-34a has previously been identified to be frequently aberrantly expressed in human cancer, and involved in the regulation of cancer cells as a tumor suppressor (13). A previous 
$\mathbf{A}$

\begin{tabular}{|c|c|}
\hline $\begin{array}{l}\text { Position } 424-431 \text { of E2F5 3' UTR } \\
\text { hsa-miR-34a-5p }\end{array}$ & 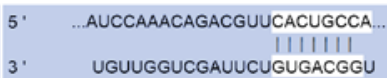 \\
\hline
\end{tabular}

B
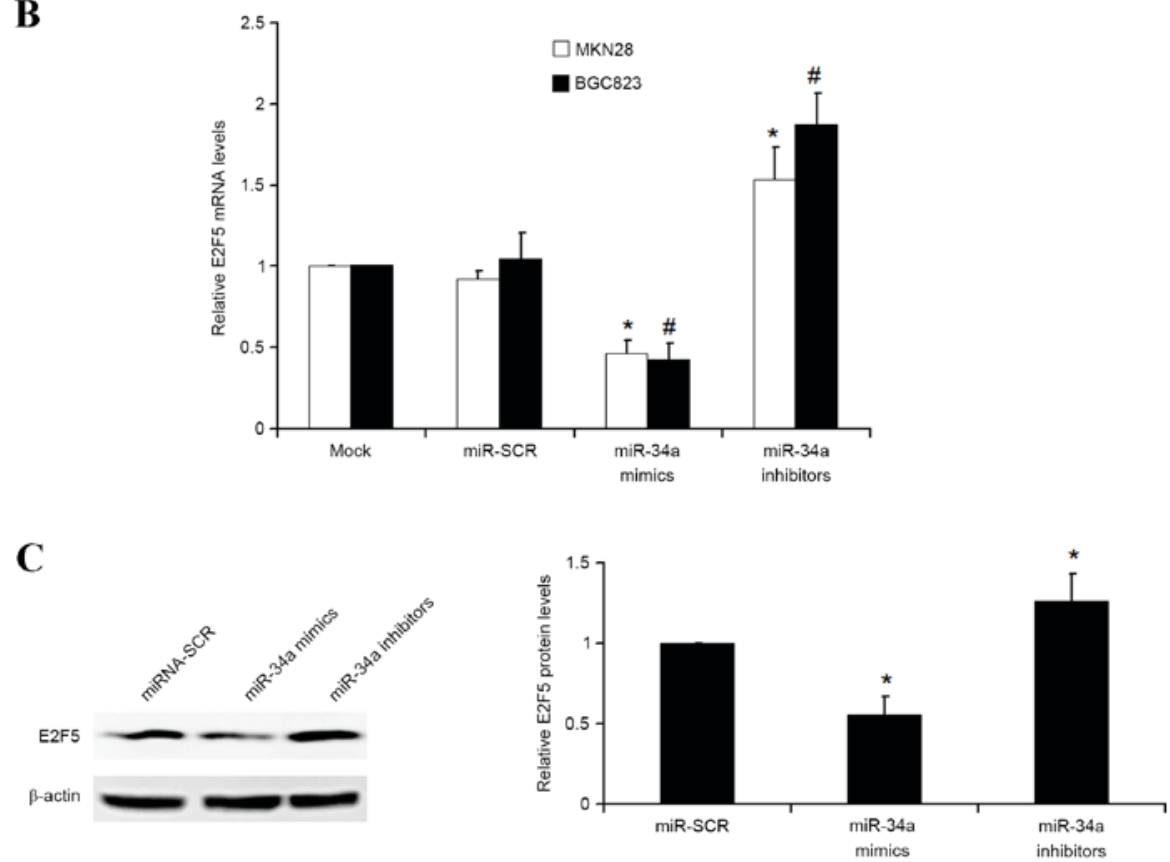

D

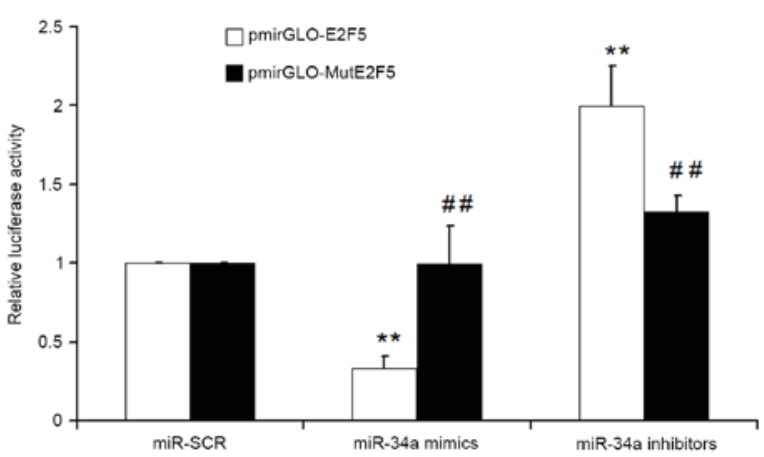

Figure 3. E2F5 is directly targeted by miR-34a. (A) TargetScan software was used to predict the potential miR-34a binding site in the 3'UTR of E2F5. (B) Quantitative polymerase chain reaction was used to determine E2F5 mRNA levels in MKN28 and BGC823 cells transfected with miR-SCR or miR-34a mimics or miR-34a inhibitors. (C) Representative western blot analysis and quantitative analysis demonstrated that E2F5 protein levels were decreased and increased by miR-34a mimics and miR-34a inhibitors, respectively. $\beta$-actin was used as the control. (D) Dual-luciferase assays were used to detect the luciferase activity in MKN28 cells transfected with pmirGLO-E2F5 or pmirGLO-MutE2F5 in the presence of miR-SCR or miR-34a mimics or miR-34a inhibitors. ${ }^{*} \mathrm{P}<0.05$ vs. miR-SCR transfected MKN28 cells; ${ }^{*} \mathrm{P}<0.05$ vs. miR-SCR transfected BGC 823 cells. ${ }^{* *} \mathrm{P}<0.05$ vs. miR-SCR+pmirGLO-E2F5 transfected MKN28 cells; ${ }^{\# \text { P }}<0.05$ compared with pmirGLO-E2F5 group. miR, microRNA; UTR, untranslated region; SCR, scrambled; Mut, mutant.

A

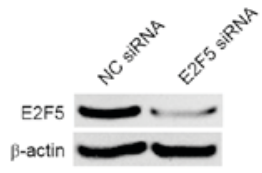

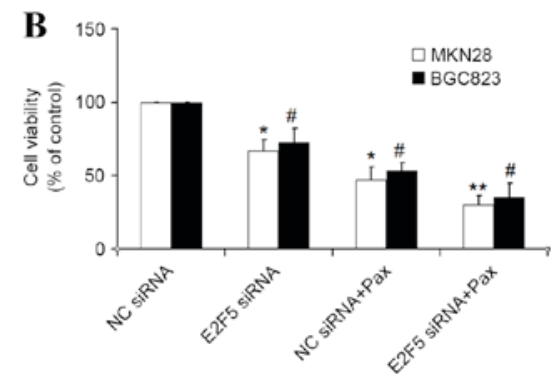

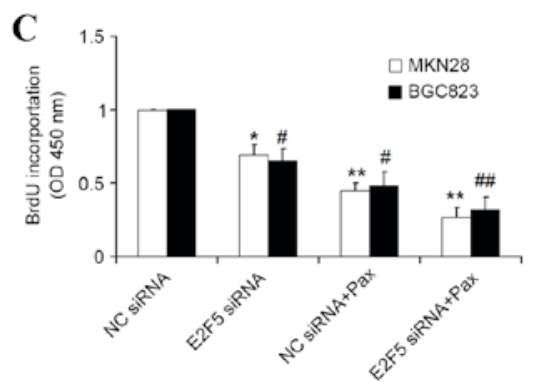

Figure 4. E2F5 knockdown by specific siRNA in GC enhanced the inhibitory effect of paclitaxel on cellular proliferation. (A) Western blot analysis was used to detect E2F5 protein levels in MKN28 cells transfected with NC siRNA or E2F5 siRNA. $\beta$-actin was used as the control (B) MTT assays were used to determine the cellular viability in MKN28 and BGC823 cells transfected with NC siRNA or E2F5 siRNA for $24 \mathrm{~h}$, followed by treatment of paclitaxel (100 nM) for another 16 h. (C) BrdU incorporation was used to detect the cellular proliferation in MKN28 and BGC823 cells transfected with miR-SCR or miR-34a for $24 \mathrm{~h}$, followed by treatment of paclitaxel $(100 \mathrm{nM})$ for another $16 \mathrm{~h} .{ }^{*} \mathrm{P}<0.05,{ }^{* *} \mathrm{P}<0.01 \mathrm{vs}$. NC siRNA transfected MKN28 cells; ${ }^{*} \mathrm{P}<0.05,{ }^{\# \#} \mathrm{P}<0.01 \mathrm{vs}$. NC siRNA transfected BGC823 cells. si, small interfering; GC, gastric cancer; NC, negative control; BrdU, 5-Bromo-2-deoxyUridine; SCR, scrambled; Pax, paclitaxel; OD, optical density. 
study identified that miRNA-34a was significantly downregulated in gastric cancer cells, and that miRNA-34a suppressed EGFR-dependent MMP-7 activation (14). Additionally, another study demonstrated that miRNA-34a was able to regulate the proliferation, invasion and migration of GC cells by targeting survivin (23), an important anti-apoptotic gene (24). Consistent with these studies, the present results identified that miRNA-34a was able to inhibit the growth rate of GC compared with scrambled miRNA. A recent study suggested that miRNA-34a-5p can enhance chemotherapeutic sensitivity to cisplatin in hepatocellular carcinoma MHCC-97 L cells (25). However, whether miRNA-34a enhances the chemotherapeutic efficacy of paclitaxel remains unknown. The present study identified that miRNA-34a transfection could significantly enhance the inhibitory effect of paclitaxel on the cellular proliferation of GC, suggesting a potential role of miRNA-34a in treatment of GC combined with paclitaxel. However, the present study did not confirm whether miRNA-34a is capable of enhancing the role of paclitaxel in the inhibition of migration and invasion of GC cells. Therefore, future investigation is warranted.

The present results demonstrated that miRNA-34a was able to negatively regulate E2F5 by binding to the conserved miRNA-34a binding site in the 3'UTR of E2F5. The transcription factor E2F performs a central role in cell growth and proliferation by controlling cell cycle (26), which suggests that E2F5 may also be involved in cellular proliferation and cell cycle progression as an important member of the E2F family. Previous data has reported that E2F5 was overexpressed in various types of human cancer including breast, epithelial ovarian and prostate cancer and hepatocellular carcinoma, and closely associated with cancer progression and prognosis (16-19). However, the role of E2F5 in GC remained unclear. The present study identified that E2F5 knockdown by specific siRNA exerted a significant inhibitory effect on the growth rate of GC cells, indicating that E2F5 may serve as a key oncogene in GC. Importantly, E2F5 knockdown combined with paclitaxel exhibited an increased inhibitory profile compared with treatment with E2F5 siRNA or paclitaxel alone, which may provide a more promising chemotherapeutic strategy for GC. Therefore, E2F5 may be an important target for treatment of GC. However, whether E2F5 is also implicated in cell migration, invasion and apoptosis in GC is unclear, and requires additional investigation.

In conclusion, the present study demonstrated the role of miRNA-34a and its target E2F5 in the chemotherapeutic sensitive of paclitaxel, which will not only aid future elucidation of function of miRNA-34a/E2F5 axis in various cancers, but also assist in the development of a promising chemotherapeutic strategy for the treatment of GC.

\section{References}

1. Forman D and Burley VJ: Gastric cancer: Global pattern of the disease and an overview of environmental risk factors. Best Pract Res Clin Gastroenterol 20: 633-649, 2006.

2. Ueda S, Kuji I, Shigekawa T, Takeuchi H, Sano H, Hirokawa E, Shimada H, Suzuki H, Oda M, Osaki A and Saeki T: Optical imaging for monitoring tumor oxygenation response after initiation of single-agent bevacizumab followed by cytotoxic chemotherapy in breast cancer patients. PLoS One 9: e98715, 2014.
3. Mita M, Gordon M, Rosen L, Kapoor N, Choy G, Redkar S, Taverna P, Oganesian A, Sahai A, Azab M, et al: Phase 1B study of amuvatinib in combination with five standard cancer therapies in adults with advanced solid tumors. Cancer Chemother Pharmacol 74: 195-204, 2014.

4. Le XF and Bast RC Jr: Src family kinases and paclitaxel sensitivity. Cancer Biol Ther 12: 260-269, 2011.

5. Wu G, Qin XQ, Guo JJ, Li TY and Chen JH: AKT/ERK activation is associated with gastric cancer cell resistance to paclitaxel. Int J Clin Exp Pathol 7: 1449-1458, 2014.

6. Zanetta G, Fei F and Mangioni C: Chemotherapy with paclitaxel, ifosfamide, and cisplatin for the treatment of squamous cell cervical cancer: The experience of Monza. Semin Oncol 27 (1 Suppl 1): S23-S27, 2000.

7. Murray S, Briasoulis E, Linardou H, Bafaloukos D and Papadimitriou C: Taxane resistance in breast cancer: Mechanisms, predictive biomarkers and circumvention strategies. Cancer Treat Rev 38: 890-903, 2012.

8. Bartel DP: MicroRNAs: Genomics, biogenesis, mechanism, and function. Cell 116: 281-297, 2004.

9. Jiang $\mathrm{C}$, Chen X, Alattar M, Wei J and Liu H: MicroRNAs in tumorigenesis, metastasis, diagnosis and prognosis of gastric cancer. Cancer Gene Ther 22: 291-301, 2015.

10. Tutar L, Tutar E, Özgür A and Tutar Y: Therapeutic targeting of microRNAs in cancer: Future perspectives. Drug Dev Res 76: 382-388, 2015.

11. Liu X, Li J, Qin F and Dai S: miR-152 as a tumor suppressor microRNA: Target recognition and regulation in cancer. Oncol Lett 11: 3911-3916, 2016.

12. Naidu S, Magee P and Garofalo M: MiRNA-based therapeutic intervention of cancer. J Hematol Oncol 8: 68, 2015.

13. Misso G, Di Martino MT, De Rosa G, Farooqi AA, Lombardi A, Campani V, Zarone MR, Gullà $\mathrm{A}$, Tagliaferri $\mathrm{P}$, Tassone $\mathrm{P}$ and Caraglia M: Mir-34: A new weapon against cancer? Mol Ther Nucleic Acids 3: e194, 2014.

14. Liu G, Jiang C, Li D, Wang R and Wang W: MiRNA-34a inhibits EGFR-signaling-dependent MMP7 activation in gastric cancer. Tumour Biol 35: 9801-9806, 2014.

15. Livak KJ and Schmittgen TD: Analysis of relative gene expression data using real-time quantitative PCR and the 2(-Delta Delta C(T)) method. Methods 25: 402-408, 2001.

16. Polanowska J, Le Cam L, Orsetti B, Vallés H, Fabbrizio E, Fajas L, Taviaux S, Theillet C and Sardet C: Human E2F5 gene is oncogenic in primary rodent cells and is amplified in human breast tumors. Genes Chromosomes Cancer 28: 126-130, 2000.

17. Kothandaraman N, Bajic VB, Brendan PN, Huak CY, Keow PB, Razvi K, Salto-Tellez M and Choolani M: E2F5 status significantly improves malignancy diagnosis of epithelial ovarian cancer. BMC Cancer 10: 64, 2010.

18. Zhao J, Wu XY, Ling XH, Lin ZY, Fu X, Deng YH, He HC and Zhong W: Analysis of genetic aberrations on chromosomal region 8q21-24 identifies E2F5 as an oncogene with copy number gain in prostate cancer. Med Oncol 30: 465, 2013.

19. Jiang Y, Yim SH, Xu HD, Jung SH, Yang SY, Hu HJ, Jung CK and Chung YJ: A potential oncogenic role of the commonly observed E2F5 overexpression in hepatocellular carcinoma. World J Gastroenterol 17: 470-477, 2011.

20. Macfarlane LA and Murphy PR: MicroRNA: Biogenesis, function and role in cancer. Curr Genomics 11: 537-561, 2010.

21. Zhang J, Li S, Li L, Li M, Guo C, Yao J and Mi S: Exosome and exosomal microRNA: Trafficking, sorting, and function. Genomics Proteomics Bioinformatics 13: 17-24, 2015.

22. Chen CZ: MicroRNAs as oncogenes and tumor suppressors. $\mathrm{N}$ Engl J Med 353: 1768-1771, 2005.

23. Cao W, Fan R, Wang L, Cheng S, Li H, Jiang J, Geng M, Jin Y and $\mathrm{Wu}$ Y: Expression and regulatory function of miRNA-34a in targeting survivin in gastric cancer cells. Tumour Biol 34: 963-971, 2013.

24. McKenzie JA and Grossman D: Role of the apoptotic and mitotic regulator survivin in melanoma. Anticancer Res 32: 397-404, 2012.

25. Li XY, Wen JY, Jia CC, Wang TT, Li X, Dong M, Lin QU, Chen ZH, Ma XK, Wei LI, et al: MicroRNA-34a-5p enhances sensitivity to chemotherapy by targeting AXL in hepatocellular carcinoma MHCC-97L cells. Oncol Lett 10: 2691-2698, 2015.

26. Chen HZ, Tsai SY and Leone G: Emerging roles of E2Fs in cancer: An exit from cell cycle control. Nat Rev Cancer 9: 785-797, 2009. 